\title{
The Vicorder device compared with SphygmoCor in the assessment of carotid-femoral pulse wave velocity in patients with peripheral arterial disease
}

\author{
Yousef Shahin, Hashem Barakat, Rachel Barnes and Ian Chetter
}

To assess the reliability and reproducibility of the Vicorder's carotid-femoral pulse wave velocity (cfPWV) measurements in patients with peripheral arterial disease (PAD) and to compare between cfPWV measurements obtained using the Vicorder with those obtained using the SphygmoCor device as a reference. Some 30 patients with PAD (23 men, mean age 64.9 \pm 7.5 ) underwent cfPWV measurement twice by a single investigator during one visit using the Vicorder and the SphygmoCor according to the manufacturer's instructions. Intra-rater reproducibility for each device was assessed using intraclass correlation coefficients (ICC) and Bland-Altman method. The latter was also used to compare between the two devices. The mean difference (s.d.) between repeated measurements was $0.03 \pm 0.92 \mathrm{~m} \mathrm{~s}^{-1}, P=0.85$ and $0.01 \pm 0.54 \mathrm{~m} \mathrm{~s}^{-1}, P=0.91$ for the SphygmoCor and Vicorder, respectively. Measurements of cfPWV were highly reproducible using both devices (ICC $=0.94$ and 0.92, for the Vicorder and SphygmoCor, respectively). Limits of Agreement using the Bland-Altman method were -1.07 to $1.09 \mathrm{~m} \mathrm{~s}^{-1}$ and -1.79 to $1.85 \mathrm{~m} \mathrm{~s}^{-1}$ for the Vicorder and the SphygmoCor, respectively. Bland-Altman plots indicated that $90 \%$ of the cfPWV measurements using the Vicorder and $93 \%$ of the measurements using the SphygmoCor fell within two s.d.s of the mean difference. Transit time (TT) differed significantly between the two devices (mean difference $30 \pm 9.2 \mathrm{~m} \mathrm{~s}$, $P<0.001$ ), with the Vicorder recording higher values. Nevertheless, the two devices recorded nearly similar cfPWV measurements (mean difference $-0.69 \pm 1.6 \mathrm{~m} \mathrm{~s}^{-1}, P=0.02$ ) with $97 \%$ of cfPWV values falling within two s.d. values of the mean difference on Bland-Altman plot. Both devices generated highly reproducible cfPWV measurements in patients with PAD and were in good agreement when compared with each other. However, the discrepancy in TT between the two devices lead to the Vicorder producing lower values of cfPWV at high values produced by the SphygmoCor.

Hypertension Research (2013) 36, 208-212; doi:10.1038/hr.2012.144; published online 4 October 2012

Keywords: arterial stiffness; peripheral arterial disease; pulse wave velocity; sphygmoCor; vicorder device

\section{INTRODUCTION}

Arterial stiffness assessed by cfPWV is associated with several pathological conditions ${ }^{1-4}$ and has been identified as a risk factor for cardiovascular disease. ${ }^{5}$ This indirect measurement of arterial stiffness is considered the gold standard of PWV measurement, ${ }^{6}$ and is based on estimating the velocity of the pressure wave travelling between the carotid and femoral arterial sites. Velocity is determined by calculating the transit time (TT) based on foot-to-foot methodology and by measuring the distance between the carotid and femoral recording sites along the body surface using a tape measure.

Several devices are available in the market for PWV measurement including the following: the SphygmoCor (AtCor Medical, Sydney, Australia), the Complior (Artech Medical, Pantin, France), the PulsePen (DiaTence, Milan, Italy) and, most recently, the Vicorder (Skidmore Medical, Bristol, UK). The SphygmoCor system uses sequential applanation tonometry of the arterial waveform with electrocardiogram gating. It is easily applied and has been widely used in adults with excellent reproducibility. ${ }^{7,8}$ The Complior system, on the other hand, uses mechanotransducers to detect the pressure waveforms and allows simultaneous recordings. The latter two devices have shown difference in PWV values when compared with each other mainly because of differences in the methods used to measure TT rather than in the methods used to acquire the waveforms. ${ }^{9,10}$ Nevertheless, to date, there is no agreement to which device is more accurate in terms of PWV measurement. ${ }^{11}$

The most recent device used to measure PWV to enter the market is the Vicorder. This device uses oscillometric technique to detect the pulse waveform between the two recording sites. It is considered to be less time-consuming and less operator-skill dependent. The reproducibility of the Vicorder has been assessed in comparison with the more established SphygmoCor system in healthy subjects ${ }^{9,12}$ and in 
children. ${ }^{13,14}$ However, to date, there are no studies in the literature comparing between the two devices in the context of peripheral arterial disease (PAD). Patients with $\mathrm{PAD}$ have increased arterial stiffness and difficult-to-palpate peripheral pulses. Thus, this study objectives were to assess the reproducibility of the Vicorder device in patients with PAD and to compare between cfPWV measurements obtained using the Vicorder with those obtained using the SphygmoCor as a reference.

\section{METHODS}

This study was conducted in accordance with the declaration of Helsinki and the international conference for harmonisation and good clinical practice (ICH/GCP) guidelines. Following approval from the local research ethics committee and the hospital research and development department, patients diagnosed with symptomatic lower limb arterial disease or intermittent claudication were prospectively recruited from a vascular consultant-led outpatient clinic of a university teaching hospital over a 10-month period from February 2011. All recruited patients underwent initial assessments, which included a thorough clinical history and examination, and establishing current smoking status. Heart rate, systolic blood pressure, diastolic blood pressure and body mass index were determined. Blood pressure (BP) was measured using an automated oscillometric device (Welch Allyn, Arden, NC, USA). This device has been validated in terms of BP measurement in several previous studies. ${ }^{15-17}$ All patients underwent ankle brachial pressure index (ABPI) measurement of each leg (ABPI of the most symptomatic leg was recorded) and an arterial duplex ultrasound of the affected leg to confirm the diagnosis of intermittent claudication. Patients with an ABPI $<0.9$ with evidence of arterial disease of the lower limbs on arterial duplex ultrasound were included in the study.

\section{cfPWV measurement}

A group of 30 patients with a confirmed diagnosis of intermittent claudication underwent cfPWV measurement twice, using both the SphygmoCor and Vicorder devices, in a single visit by a single investigator (YS). Measurements were performed according to the manufacturer's manual of each device. Patients rested in a supine position for $10 \mathrm{~min}$ before the measurement in a quiet room. The sequence of cfPWV measurements was SphygmoCor 1, Vicorder 1, SphygmoCor 2 and Vicorder 2.

SphygmoCor. The SphygmoCor (Model SCOR-Pvx, software version 8; AtCor Medical) was used for cfPWV measurements. It uses the applanation tonometry technique to acquire the pulse waveform using one tonometric Miller transducer (Miller Instruments Inc., Houston, TX, USA). To obtain the cfPWV, the carotid and femoral pulse waveforms are recorded sequentially using the transducer and at the same time an electrocardiogram is recorded as a reference to calculate TT using the foot-to-foot method. The distance the pulse waveform travels between the two recording sites (carotid and femoral) is measured using a tape measure over the body area. The distance between the suprasternal notch and the carotid recording site (proximal) as well as the distance between the suprasternal notch and the femoral recording site (distal) are measured. The difference between the proximal and the distal distances is then calculated automatically by the device upon entering the former distances. The cfPWV is then calculated as cfPWV $\left(\mathrm{m} \mathrm{s}^{-1}\right)=$ distance $(\mathrm{m}) / \mathrm{TT}(\mathrm{s}){ }^{7,9}$ All measurements acquired in this study by the SphygmoCor had an operator index $>90 \%$.

Vicorder. The Vicorder (software version 4; Skidmore Medical) was used for cfPWV measurement. This is a relatively new device, which uses the oscillometric technique to acquire the pulse waveform. Measurements of cfPWV were obtained by using a $10-\mathrm{cm}$-wide cuff around the right upper thigh to detect the femoral pulse and a $3-\mathrm{cm}$ cuff around the neck to detect the right carotid pulse. The neck cuff is designed in a way that it is placed over one carotid artery to avoid compression of the trachea and both carotid arteries at the same time. The distance between the suprasternal notch and mid upper thigh cuff was used according to the manufacturer's instructions. The cuffs were automatically inflated simultaneously to $65 \mathrm{~mm} \mathrm{Hg}$ and pulse waveforms were recorded for $3-5 \mathrm{~s}$, while the patient was in supine position before freezing the display screen and obtaining cfPWV and TT.

\section{Statistical analysis}

A Statistical Package for the Social Sciences Program version 18 for Windows (SPSS, Chicago, IL, USA) was used for statistical analysis. Continuous variables were expressed as mean \pm s.d. for parametric variables and median (interquartile range) for nonparametric variables. Intra-rater reproducibility for each device was assessed using the Bland-Altman method, ${ }^{15}$ the intraclass correlation coefficient and the coefficient of variation. However, coefficient of variation can be a misleading measure of variability if values are close to zero and can unnecessarily indicate poor reproducibility. ${ }^{18}$ The values of cfPWV obtained by the two devices were compared using the Bland-Altman $\operatorname{plot}^{19}$ and Student's paired $t$-test, which was also used to compare between the TT values of two devices and distances between the two recording sites. In Bland-Altman plot, the mean value of two measurements from each patient is plotted against the difference between the two measurements. To rule out a systematic difference between two measurements, the $95 \%$ confidence interval of the mean difference should include the zero value. The correlation between the values of two devices was assessed using Pearson's correlation coefficient $(r)$ and linear regression. A $P$ value of $<0.05$ was considered to be statistically significant. All tests were two-tailed.

\section{RESULTS}

Patients' characteristics are presented in Table 1. Overall, there were more males than females ( 23 vs. 7 , respectively). The mean difference \pm s.d. between repeated cfPWV measurements was $0.03 \pm 0.92 \mathrm{~m} \mathrm{~s}^{-1}$, $P=0.85$ and $0.01 \pm 0.54 \mathrm{~m} \mathrm{~s}^{-1}, P=0.91$ for the SphygmoCor and Vicorder devices, respectively. cfPWV measurements were highly reproducible using both devices (intraclass correlation coefficients $=0.94$ and 0.92 for the Vicorder and SphygmoCor, respectively). The SphygmoCor had a lower coefficient of variation than the Vicorder; $5 \%$ vs. $6 \%$, respectively. Limits of agreement between the Vicorder repeated measurements were -1.07 to $1.09 \mathrm{~m} \mathrm{~s}^{-1}$ and -1.79 to $1.85 \mathrm{~m} \mathrm{~s}^{-1}$ for the SphygmoCor. BlandAltman plots indicated that $90 \%$ of the cfPWV measurements using the Vicorder (Figure 1) and $93 \%$ of the measurements using

\section{Table 1 Summary of patients' characteristics}

\section{Characteristics}

Age (years)

$64.9 \pm 7.5^{a}$

Height $(\mathrm{cm})$

$168+7.7$

Weight (kg)

$80.6 \pm 13.6^{a}$

BMI $\left(\mathrm{kg} \mathrm{m}^{-2}\right)$

$28.4 \pm 4^{\mathrm{a}}$

Heart rate (bpm)

$68.7 \pm 9.6^{a}$

Systolic BP ( $\mathrm{mm} \mathrm{Hg}$ )

$38(130-157)^{\mathrm{b}}$

Diastolic BP $(\mathrm{mm} \mathrm{Hg})$

$80(74-83)^{\mathrm{b}}$

Mean BP $(\mathrm{mm} \mathrm{Hg})$

$98.6(94-107)^{b}$

Pulse pressure $(\mathrm{mm} \mathrm{Hg})$

$60(50-77)^{\mathrm{b}}$

ABP

$0.62 \pm 0.19^{a}$

50

Current smokers (\%)

10

$\begin{array}{ll}\text { Medications } & \\ \text { Aspirin (\%) } & 83 \\ \text { Statins (\%) } & 80 \\ \text { Calcium channel blockers (\%) } & 43 \\ \beta \text {-blockers (\%) } & 13\end{array}$

Abbreviations: ABPI, ankle brachial pressure index; BMI, body mass index; BP, blood pressure. a Mean \pm s.d.

'Median (interquartile range) 


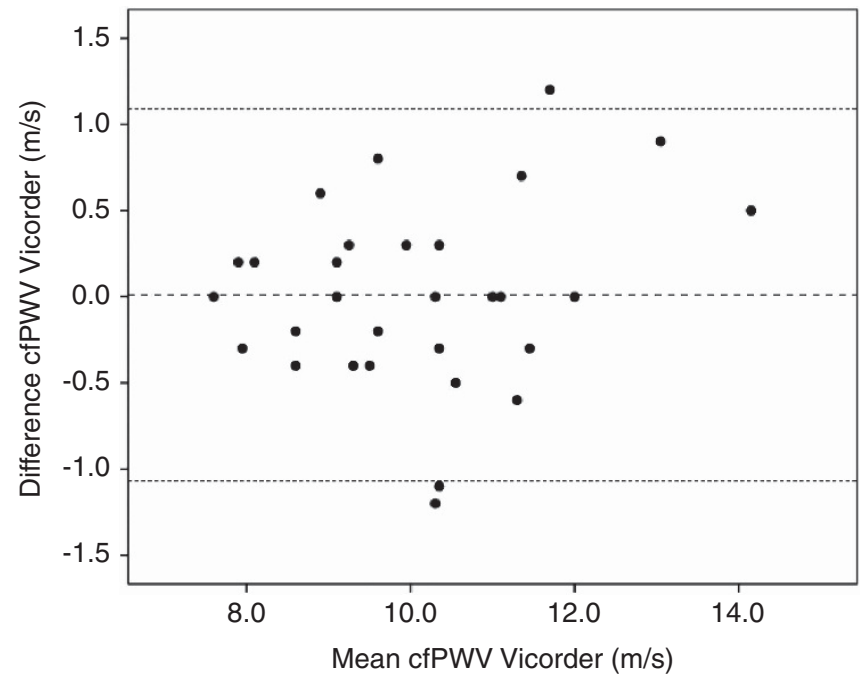

Figure 1 Bland-Altman plot illustrating intra-rater reproducibility of the cfPWV measurements by the Vicorder device. The upper and lower dotted lines represent the limits of agreement between repeated cfPWV measurements (mean difference \pm 2 s.d.s). The middle dotted line represents the mean of the difference between all repeated cfPWV measurements generated by the device.

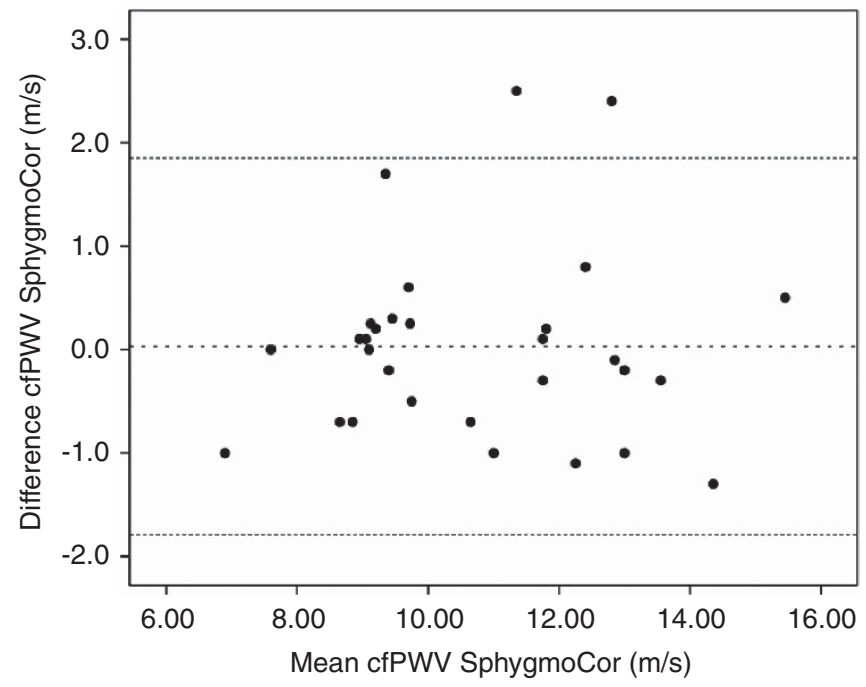

Figure 2 Bland-Altman plot illustrating intra-rater reproducibility of the cfPWV measurements by the SphygmoCor device. For further explanation, see legend for Figure 1.

the SphygmoCor (Figure 2) fell within 2 s.d. values of the mean difference.

The distance between the two recording sites (carotid-femoral) differed significantly between the two devices with longer distances associated with the Vicorder than the SphygmoCor $(0.76 \pm 0.05$ vs. $0.49 \pm 0.04 \mathrm{~m}$, respectively, mean difference $-0.26 \pm 0.05 \mathrm{~m}, P<0.001$ ). As a result, the pulse waveform TT values differed between the two devices with the Vicorder recording higher values than the SphygmoCor $(77.3 \pm 13.6$ vs. $47.2 \pm 9.9 \mathrm{~m} \mathrm{~s}$, respectively, mean difference $30 \pm 9.2 \mathrm{~m} \mathrm{~s}, P<0.001)$. However, TT values recorded by the two devices significantly positively correlated using Pearson's correlation, showing a linear relationship $(r=0.74, \quad P<0.001)$ (Figure 3).

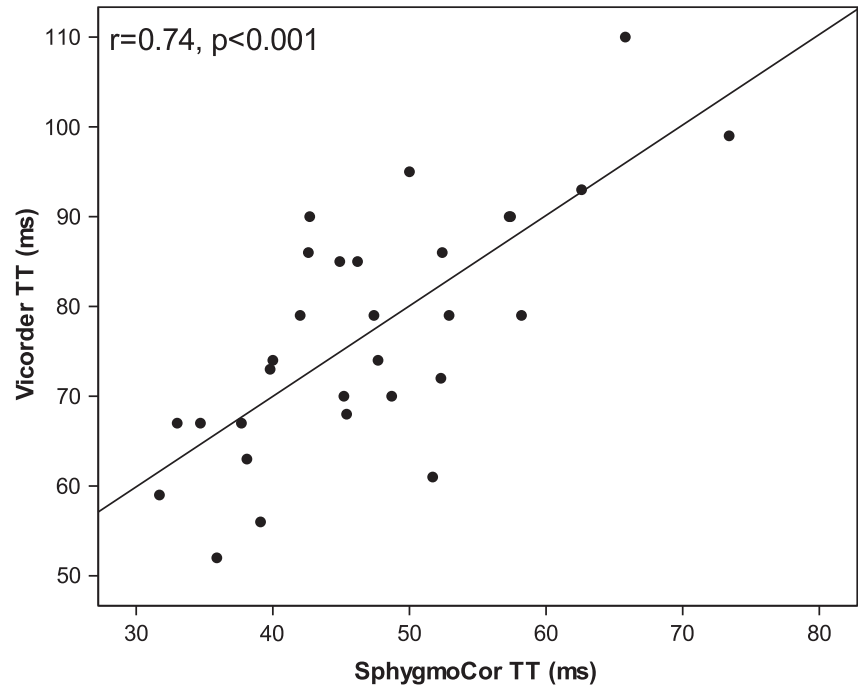

Figure 3 Scatter plot illustrating the relationship between TT values obtained by the SphygmoCor with those obtained by the Vicorder. The black line is the regression line.

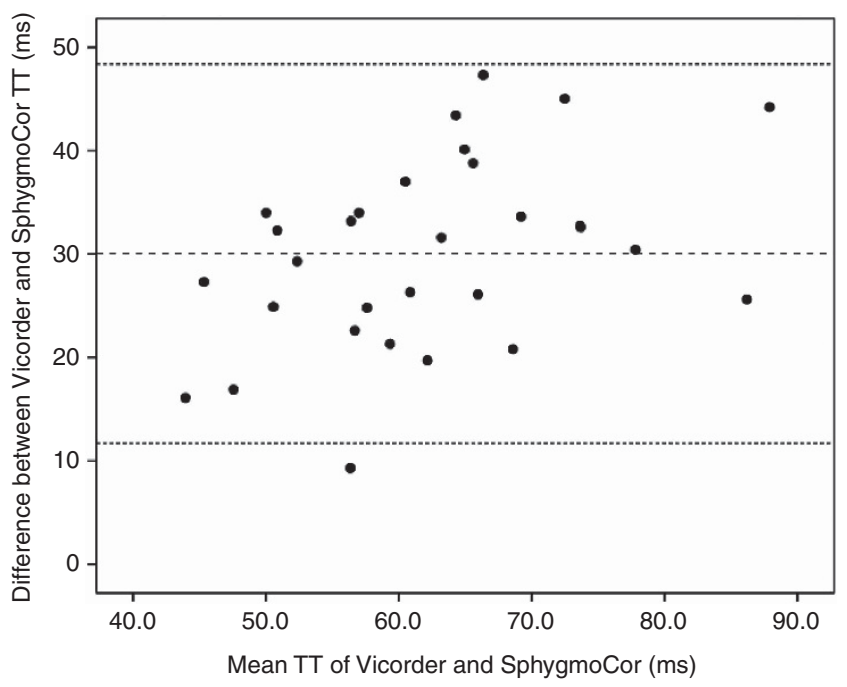

Figure 4 Bland-Altman plot illustrating the agreement in TT values between the SphygmoCor and the Vicorder. For further explanation see legend for Figure 1.

Although both devices showed good linear agreement, this was not the case using Bland-Altman plot, where limits of agreement between the two devices TT values were $11.72-48.36 \mathrm{~m} \mathrm{~s}$, which indicated a systematic difference in TT values. (Figure 4).

The mean difference between cfPWV values obtained by the two devices was statistically significant $-0.69 \pm 1.6 \mathrm{~m} \mathrm{~s}^{-1}, P=0.02$, with the SphygmoCor recording higher values than the Vicorder $\left(10.77 \pm 1.6 \mathrm{~m} \mathrm{~s}^{-1}\right.$ vs. $10.08 \pm 2.1 \mathrm{~m} \mathrm{~s}^{-1}$, respectively). Nevertheless, cfPWV measurements showed a linear relationship with strong correlation ( $r=0.67, P<0.001$; Figure 5) and using Bland-Altman Plot, the limits of agreement between the two devices measurements were 2.53 to $-3.91 \mathrm{~m} \mathrm{~s}^{-1}$ with $97 \%$ of the cfPWV measurements falling 


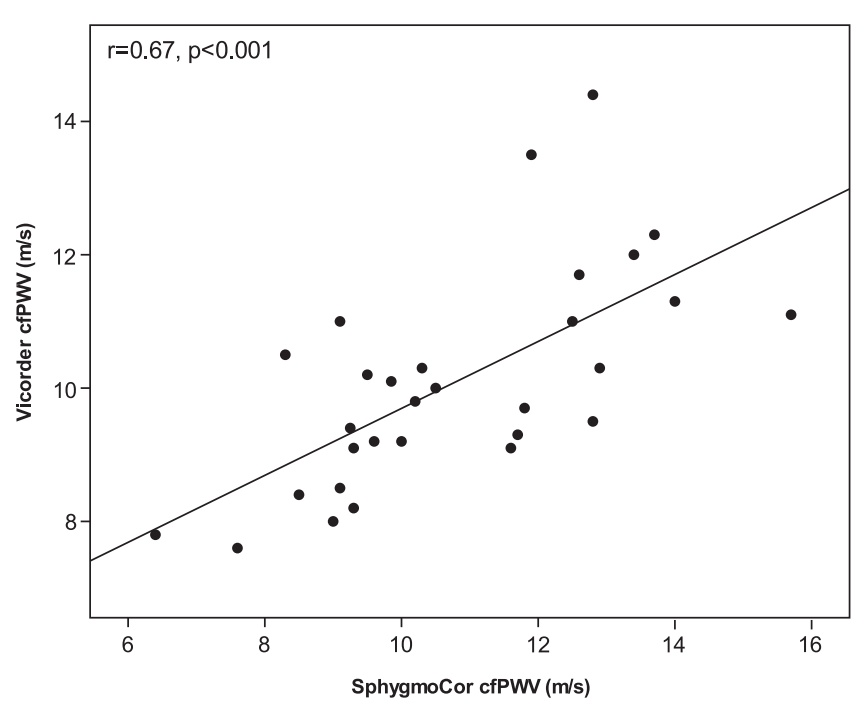

Figure 5 Scatter plot illustrating the relationship between cfPWV values obtained by the SphygmoCor and those obtained by the Vicorder. The black line is the regression line.

within 2 s.d.s of the mean difference. Interestingly, however, the Vicorder device tended to generate lower values of cfPWV at high values of cfPWV generated by the SphygmoCor mainly at cfPWV values $>10 \mathrm{~m} \mathrm{~s}^{-1}$ (Figure 6).

\section{DISCUSSION}

Previous studies have assessed the reliability and reproducibility of the Vicorder device in comparison with the gold standard SphygmoCor system in the measurement of cfPWV in healthy subjects ${ }^{9,12}$ and in children. ${ }^{13,14}$ However, this is the first study in the literature to compare between the two device's cfPWV values in patients with PAD. The difference between healthy subjects and patients with PAD is that the latter have higher values of cfPWV owing to increased arterial stiffness caused by atherosclerosis. This might have a role in the increased cardiovascular morbidity and mortality associated with PAD. Therefore, assessment of arterial stiffness in this patients' cohort can provide valuable prognostic information. Hence, it is imperative to find less operator-skill dependent and less time-consuming devices that can assess cfPWV. In addition, in patients with PAD, peripheral pulses are harder to record in comparison with healthy subjects because of stenotic arteries. We encountered this problem using the SphygmoCor while trying to acquire high-quality recordings of the femoral pulse. This is because most of our study subjects had atherosclerotic disease of the femoral artery. However, this problem was not encountered with the Vicorder, as the device was able to produce high-quality recordings of the femoral pulse waveform.

The main finding of this study is that the Vicorder and SphygmoCor cfPWV values were in good agreement, although the Vicorder device differed significantly from SphygmoCor in terms of giving higher TT values and longer distances between the two recording sites measured, according to the manufacturer's instructions. This can be explained by the difference in the transit distance measurement technique between the two devices, as the distance between the carotid pulse detection point and the suprasternal notch is not substracted from the distance between the suprasternal notch and the distal pulse detection point (femoral), in the case of the Vicorder compared with SphygmoCor. As a result of the increased distance, it is self-evident that the TT values will be higher in the case of Vicorder

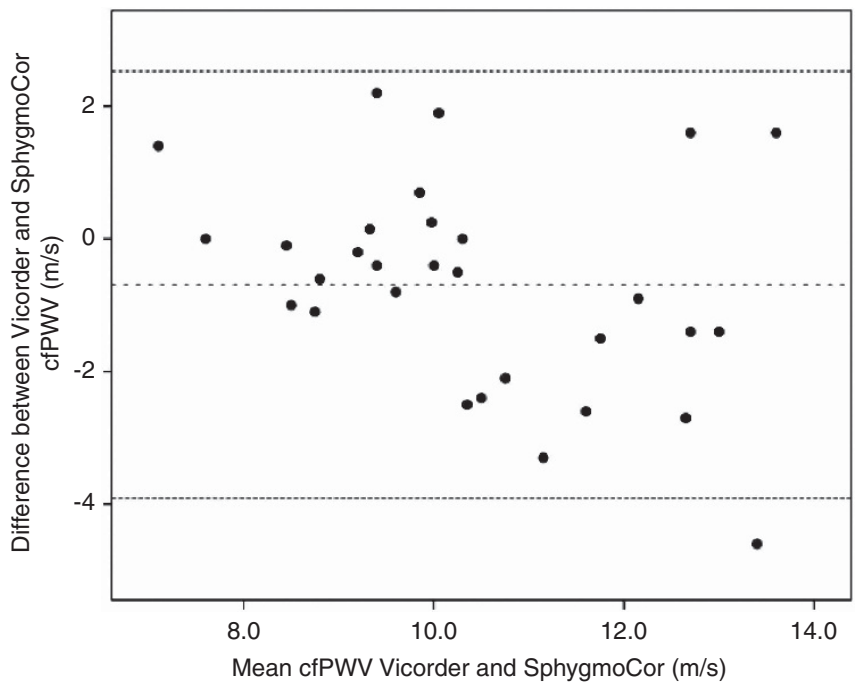

Figure 6 Bland-Altman plot illustrating the agreement between cfPWV measurements obtained by the SphygmoCor and those obtained by the Vicorder. For further explanation, see legend for Figure 1.

compared with SphygmoCor. Despite this discrepancy in transit distance and TT values between the two devices, there is a good agreement between cfPWV measurements acquired by both devices, which can be explained by the fact that cfPWV is a ratio between transit distance and time; so when transit distance is divided by TT, the higher values of both measures cancel out and the ratio remains constant. Nevertheless, the Vicorder values tended to produce lower cfPWV values than the SphygmoCor at high arterial stiffness values (at values $>10 \mathrm{~m} \mathrm{~s}^{-1}$ ), resulting in a statistically significant mean difference in cfPWV measurements of $0.69 \mathrm{~m} \mathrm{~s}^{-1}$ between the two devices with the SphygmoCor recording higher cfPWV values than the Vicorder. However, we are not sure whether this mean difference between the two devices is of any clinical significance. A previous study ${ }^{9}$ attributed this difference to the discrepancy in TT and transit distance values between the two devices. In Hickson et al., ${ }^{9}$ the distance between the two recording sites using the Vicorder was measured differently in comparison with our study. The authors measured a path length from the suprasternal notch to the top of the thigh cuff. They further implemented an adjustment for the distance to the pulse detection point in the thigh cuff, which brought cfPWV values from both devices to good agreement with a mean difference of $0.31 \mathrm{~m} \mathrm{~s}^{-1}, P<0.001$. However, authors encountered the same problem of the Vicorder reporting lower cfPWV values than the SphygmoCor at high values of arterial stiffness. In another study, ${ }^{12}$ the distance was measured as the difference between the distance between the upper edge of the femoral cuff and sternal notch, and the distance between the lower edge of the neck cuff and sternal notch. However, although Hickson et al. ${ }^{9}$ found good agreement between cfPWV values generated by both devices, the latter study ${ }^{12}$ showed no agreement with the Vicorder recording higher values of cfPWV than the SphygmoCor (mean difference (95\% confidence interval) $\left.0.58 \mathrm{~m} \mathrm{~s}^{-1}(-0.2,1.35)\right)$. In our study, the distance was measured between the suprasternal notch and the pulse detection point at the mid-thigh cuff, according to the manufacturer's instructions. As a result, cfPWV values from both devices were in good agreement but the issue of the discrepancy between the two device's values at high arterial stiffness values persisted. Another factor that might have contributed to the discrepancy between the two device's cfPWV values 
at high arterial stiffness values could be the difference in the technique each device uses to measure cfPWV. Although the SphygmoCor uses electrocardiogram-gated sequential pressure waveform measures, the Vicorder uses simultaneous recordings. As a result, there will be variation in basal autonomic state between sequential SphygmoCor measurements, which is not encountered in the Vicorder measurements. The difference in heart rate and brachial BP recorded by the two devices can also have a role. Brachial $\mathrm{BP}$ values are entered in the SphygmoCor after being measured by a cuff-based device or manually; however, brachial BP values used by the Vicorder are measured by the device itself using an oscillometric technique. Variations in these parameters could account for the discrepancy in cfPWV measurements generated by the two devices.

The Vicorder was easy to use, needing less operator training, consuming less time and more comfortable to the patient than the SphygmoCor. It is worth mentioning that the neck cuff used to detect the carotid pulse was well tolerated by patients in spite of the fact that it was inflated up to $65 \mathrm{~mm} \mathrm{Hg}$. The SphygmoCor, on the other hand, was intrusive needing exposure of the groin to detect the femoral pulse. The Vicorder cfPWV measurements, in our study, which are in agreement with other studies, ${ }^{9}$ were highly reproducible as well as repeatable and were in good agreement with those produced by the SphygmoCor, showing strong correlation and excellent fit on BlandAltman plot.

To conclude, studies which have reported on the Vicorder's reproducibility used different methodologies in terms of distance measurement. We found no consensus on a standardized measurement method. Nevertheless, this study showed that the Vicorder generates highly reproducible cfPWV measurements, which are comparable to those generated by the SphygmoCor in patients with PAD.

\section{CONFLICT OF INTEREST}

The authors declare no conflict of interest.

\section{ACKNOWLEDGEMENTS}

We are grateful for the Welsh Heart Research Institute for providing training on using the SphygmoCor. We are also grateful to Professor JR Cockcroft for facilitating the training on the device. YS and IC received funding for a medical device (Vicorder) from the Yorkshire Vascular and Surgical Research Fund, UK.
1 Safar ME, Levy BI, Struijker-Boudier H. Current perspectives on arterial stiffness and pulse pressure in hypertension and cardiovascular diseases. Circulation 2003; 107 : 2864-2869.

2 London GM, Marchais SJ, Guerin AP, Pannier B. Arterial stiffness: pathophysiology and clinical impact. Clin Exp Hypertens 2004; 26: 689-699.

3 Laurent S, Boutouyrie P, Asmar R, Gautier I, Laloux B, Guize L, Ducimetiere P, Benetos A. Aortic stiffness is an independent predictor of all-cause and cardiovascular mortality in hypertensive patients. Hypertension 2001; 37: 1236-1241.

4 Blacher J, Guerin AP, Pannier B, Marchais SJ, Safar ME, London GM. Impact of aortic stiffness on survival in end-stage renal disease. Circulation 1999; 99: 2434-2439.

5 Arnett DK, Evans GW, Riley WA. Arterial stiffness: a new cardiovascular risk factor? Am J Epidemiol 1994; 140: 669-682.

6 Laurent S, Cockcroft J, Van Bortel L, Boutouyrie P, Giannattasio C, Hayoz D, Pannier B, Vlachopoulos C, Wilkinson I, Struijker-Boudier H. Expert consensus document on arterial stiffness: methodological issues and clinical applications. Eur Heart J 2006 27: 2588-2605.

7 Wilkinson IB, Fuchs SA, Jansen IM, Spratt JC, Murray GD, Cockcroft JR, Webb DJ. Reproducibility of pulse wave velocity and augmentation index measured by pulse wave analysis. J Hypertens 1998; 16: 2079-2084.

8 Frimodt-Moller M, Nielsen AH, Kamper AL, Strandgaard S. Reproducibility of pulsewave analysis and pulse-wave velocity determination in chronic kidney disease. Nephrol Dial Transplant 2008; 23: 594-600.

9 Hickson SS, Butlin M, Broad J, Avolio AP, Wilkinson IB, McEniery CM. Validity and repeatability of the Vicorder apparatus: a comparison with the SphygmoCor device. Hypertens Res 2009; 32: 1079-1085.

10 Millasseau SC, Stewart AD, Patel SJ, Redwood SR, Chowienczyk PJ. Evaluation of carotid-femoral pulse wave velocity: influence of timing algorithm and heart rate. Hypertension 2005; 45: 222-226.

11 Rajzer MW, Wojciechowska W, Klocek M, Palka I, Brzozowska-Kiszka M, KaweckaJaszcz K. Comparison of aortic pulse wave velocity measured by three techniques: Complior, SphygmoCor and Arteriograph. J Hypertens 2008; 26: 2001-2007.

12 van Leeuwen-Segarceanu EM, Tromp WF, Bos WJ, Vogels OJ, Groothoff JW, van der Lee $\mathrm{JH}$. Comparison of two instruments measuring carotid-femoral pulse wave velocity: Vicorder versus SphygmoCor. J Hypertens 2010; 28: 1687-1691.

13 Kis E, Cseprekal O, Kerti A, Salvi P, Benetos A, Tisler A, Szabo A, Tulassay T, Reusz GS. Measurement of pulse wave velocity in children and young adults: a comparative study using three different devices. Hypertens Res 2011; 34: 1197-1202.

14 Kracht D, Shroff R, Baig S, Doyon A, Jacobi C, Zeller R, Querfeld U, Schaefer F, Wuhl E, Schmidt BM, Melk A. Validating a new oscillometric device for aortic pulse wave velocity measurements in children and adolescents. Am J Hypertens 2011; 24 1294-1299.

15 Jones CR, Taylor K, Poston L, Shennan AH. Validation of the Welch Allyn 'Vital Signs' oscillometric blood pressure monitor. J Hum Hypertens 2001; 15: 191-195.

16 Alpert BS. Validation of the Welch Allyn Spot Vital Signs blood pressure device according to the ANSI/AAMI SP10: 2002. Accuracy and cost-efficiency successfully combined. Blood Press Monit 2007; 12: 345-347.

17 Davis PD, Dennis JL, Railton R. Evaluation of the A\&D UA-767 and Welch Allyn Spot Vital Signs noninvasive blood pressure monitors using a blood pressure simulator. I Hum Hypertens 2005; 19: 197-203.

18 Papaioannou TG, Karatzis EN, Karatzi KN, Gialafos EJ, Protogerou AD, Stamatelopoulos KS, Papamichael CM, Lekakis JP, Stefanadis CI. Hour-to-hou and week-to-week variability and reproducibility of wave reflection indices derived by aortic pulse wave analysis: implications for studies with repeated measurements. J Hypertens 2007; 25: 1678-1686.

19 Bland JM, Altman DG. Statistical methods for assessing agreement between two methods of clinical measurement. Lancet 1986; 1: 307-310. 